\title{
Salud colectiva en tiempos de COVID-19. Modelo escalar para comprender las afectaciones en la ruralidad de Urabá, Antioquia, Colombia, 2020*
}

\author{
Collective health in times of COVID-19. A scalar model \\ for understanding the impacts on rurality in Urabá, \\ Antioquia, Colombia, 2020
}

\section{Saúde coletiva em tempos da COVID-19. Modelo escalar para entender os efeitos na ruralidade de Urabá, Antioquia, Colômbia, 2020}

Recibido: 30 de Noviembre de 2020. Aceptado: 05 de Febrero de 2021.

Publicado: 30 de diciembre de 2021.

DOI: https://doi.org/10.11144/Javeriana.rgps20.sctc

Beatriz Elena Arias López ${ }^{a}$

Universidad de Antioquia, Colombia

ORCID: https://orcid.org/0000-0002-3326-0402

Eulalia Hernández Ciro

Universidad de Antioquia, Colombia

ORCID: https://orcid.org/0000-0002-4216-4017

\section{Alderid Gutiérrez Loaiza}

Universidad de Antioquia, Colombia

ORCID: https://orcid.org/0000-0002-9699-2563

Para citar este artículo Arias BE. Hernández E, Gutiérrez A. Salud colectiva en tiempos de COVID-19. Modelo escalar para comprender las afectaciones en la ruralidad de Urabá, Antioquia, Colombia, 2020. Rev Gerenc Polit Salud. 2021;20. https://doi.org/10.11144/Javeriana.rgps20.sctc

\footnotetext{
* Artículo de investigación
}

a Autora de correspondencia. Correo electrónico: beatriz.arias@udea.edu.co 


\section{Resumen}

Introducción. En el marco del Observatorio ruralidad y paz en contextos de pandemia y post-pandemia se presentan avances parciales de investigación y reflexiones de orden interdisciplinario sobre salud colectiva en la subregión del Urabá antioqueño, focalizados en contextos rurales. Objetivos. Identificar las dinámicas de integración/exclusión relacionadas con la salud colectiva en los territorios rurales de la subregión del Urabá antioqueño en el contexto de la pandemia COVID-19. Métodos. Se utilizó el modelo escalar de salud colectiva en tiempos de pandemia como herramienta para problematizar los datos epidemiológicos y las lecturas territoriales, en diálogo con las voces de lugareños y líderes de organizaciones sociales locales. Resultados. Los resultados permiten identificar la dinámica de la pandemia en clave de integración/exclusión de los municipios de la subregión, la superposición de lógicas de legalidad e ilegalidad que se dan en sus territorios, la amplificación de las afectaciones en territorios con cargas históricas de inequidad, y las grandes limitaciones para tener un nivel de gobernanza efectiva en salud. Conclusiones. La mayor limitación ha sido la ausencia de datos diferenciados para las áreas rurales, procedencia étnica y precisión en el origen del contagio. Este análisis nos lleva a afirmar que la pandemia por COVID-19 revela nuevamente los desbalances estructurales, especialmente en las zonas rurales de Colombia.

Palabras clave: Población rural, infecciones por coronavirus, salud colectiva, pandemia, percepción del tiempo, Colombia.

\section{Abstract}

Introduction. This research was developed in the framework of the Observatory on rurality and peace in pandemic and post-pandemic contexts, this paper presents advances of interdisciplinary research and reflections about collective health in the Antioquian subregion of Urabá, focused on rural contexts. Objective. To identify the integration/exclusion dynamics related to collective health in rural territories of the Antioquian subregion of Urabá, in the pandemic for COVID-19 context. Methods. The Scalar Model of Collective Health in Times of Pandemic was used as a tool for questioning epidemiological data and territorial readings, in dialogue with the voices of inhabitants and leaders of local social organizations. Results. Results allow to identify the dynamics of the pandemic in terms of integration/ exclusion of the municipalities of the sub-region; the overlapping logics of legality and illegality in the territories; the amplification of impacts in territories with historical burdens of inequality; and the great limitations to achieve an effective level of governance in health. Conclusions. The greatest limitation has been the absence of differentiated data for the rural areas, ethnic origin, and precision about the contagion origin. This analysis leads us to affirm that pandemic for COVID-19 reveals structural imbalances, especially in the rural areas of Colombia.

Keywords: Rural population, coronavirus infections, collective health, pandemic, time perception, Colombia.

\section{Resumo}

Introdução. No marco do Observatório de ruralidade e paz em contextos de pandemia e pós-pandemia apresentamse avances parciais de pesquisa e reflexões de ordem interdisciplinar sobre saúde coletiva na sub-região do Urabá de Antioquia, focalizados em contextos rurais. Objetivo. Identificar as dinâmicas de integração/exclusão relacionadas com a saúde coletiva nos territórios rurais da sub-região do Urabá de Antioquia, no contexto da pandemia por COVID-19. Métodos. Foi utilizado o Modelo escalar de saúde coletiva em tempos de pandemia como ferramenta para problematizar os dados epidemiológicos e as leituras territoriais, em diálogo com as vozes dos habitantes e líderes de organizações sociais locais. Resultados. Os resultados permitem identificar a dinâmica da pandemia na chave de integração/exclusão dos municípios da sub-região; a superposição de lógicas de legalidade e ilegalidade que acontecem nos territórios; a amplificação das afetações em territórios com cargas históricas de inequidade; e as grandes limitações de governança efetiva em saúde. Conclusões. A maior limitação tem sido a ausência de dados diferenciados para as áreas rurais, procedência étnica e precisão na origem do contágio. Esta análise leva-nos a afirmar que a pandemia por COVID-19 revela novamente os desequilíbrios estruturais, especialmente nas regiões rurais de Colômbia.

Palavras-chave: Rural population, coronavirus infections, collective health, pandemic, time perception, Colombia. 


\section{Introducción}

Dada la magnitud, procedencia y afectaciones de la pandemia por COVID-19 que marca una situación sin precedentes en el pasado inmediato se requiere comprender su complejidad más allá de los modelos convencionales de la epidemiología y la salud pública. Se requieren análisis que superen las manifestaciones individuales para atender la naturaleza histórica, social y cultural del proceso salud-enfermedad-atención-cuidado-muerte y las respuestas sociales para enfrentarlo desde lo institucional y lo comunitario $(1,2)$. Estos elementos, debatidos desde la década de 1970 en el campo de la medicina social/salud colectiva latinoamericanas, proponen acercamientos epistemológicos de orden colectivo, social, público e institucional, con el concurso de saberes aparentemente distantes, como los de la epidemiología y las ciencias sociales $(3,4)$.

Se ha considerado que la COVID-19 no es producto del azar, por el contrario, responde a una crisis civilizatoria mundial, cuya mitigación no es equilibrada desde el punto de vista social y exacerba desigualdades a distintas escalas. Esto implica que la pandemia, aunque es un hito importante, no es un punto cero en la trayectoria vital de poblaciones empobrecidas quienes traen un acumulado histórico de sufrimiento y enfermedad, amplificados en el actual contexto. Según De Sousa (5), el virus es un problema ambiental creado por una cultura destructiva que descuida su casa común, donde priman relaciones explotativas con otras formas de vida. Si bien es importante la biomedicina y los desarrollos esperados de una vacuna eficaz, lo que esta mirada sugiere es la necesidad de una perspectiva más amplia sobre los cuidados, lo que implica girar hacia los sujetos, individuales y colectivos, para reconocer cómo incorporan el fenómeno del COVID-19 en su vida cotidiana: cómo se protegen, cómo lo perciben, cómo se organizan y de qué formas la pandemia manifiesta y amplifica inequidades históricas.

Sin desconocer las contribuciones en el pensamiento contemporáneo de la pandemia (6-8), consideramos necesario aterrizar esas reflexiones en los contextos locales, poniendo en discusión la información estadística y epidemiológica, la normativa de entes gubernamentales, las lecturas territoriales y las experiencias de las comunidades. Para ello partimos de integrar el pensamiento histórico, tanto en la acción pública como en el campo de las discusiones académicas y las vivencias cotidianas.

Es necesario comprender los matices y densidades espaciotemporales de la pandemia, que no solo tienen que ver con su presente, sino que responden a un acumulado de inequidades. Hablamos de espacio-tiempos, de lo que esta pandemia nos muestra y de la construcción de estrategias que permitan sobrellevar y pensar en los tiempos venideros, quizás de post-pandemia. Estar en cuarentena tiene múltiples implicaciones para nuestra cotidianidad y la forma en que hemos entendido y vivido el mundo contemporáneo (9), son condiciones inéditas de la humanidad. Estos tiempos de pandemia no pueden entenderse sin los problemas estructurales y las condiciones precedentes, y no menos importante, considerar la incertidumbre emergente que cuestiona el porvenir. Es importante identificar momentos claves de los tiempos de pandemia, que inician con los primeros contagios y la declaratoria por la Organización Mundial de la Salud 
(OMS), que aún no sabemos hasta cuándo se va a extender, o incluso si inaugura una nueva periodización por nombrar.

En este artículo exponemos avances parciales de investigación en el marco del proyecto "Observatorio ruralidad y paz en contextos de pandemia y post-pandemia", en la subregión del Urabá antioqueño, focalizando en los contextos rurales, con sus matices, para aventurar una comprensión desde los postulados de la salud colectiva antes mencionados. El alcance del artículo, además de proponer una metodología, será interrogar los datos, ponerlos en diálogo y propiciar algunas líneas de aproximación preliminares.

\section{Materiales y métodos: modelo escalar de salud colectiva en tiempos de pandemia}

En marzo de 2020 la Universidad de Antioquia conformó grupos interdisciplinarios para aportar a la comprensión y atención de la pandemia ${ }^{1}$. Así surgió el grupo Análisis de impactos y riesgos en los territorios, el proceso de paz y otros procesos sociales, con investigadores de las Facultades de Educación, Enfermería, Ciencias Económicas, Instituto de Estudios Regionales, Seccionales de Oriente y Urabá y Corporación CEAM.

Este grupo se interesó por comprender cómo la contingencia afecta a las comunidades campesinas, afro, indígenas, anfibias, pescadores y excombatientes. Una de sus iniciativas es el desarrollo del Observatorio ruralidad y paz en contextos de pandemia y post pandemia financiado por la misma Universidad hasta junio de 2021, en el cual se pretenden indagar las afectaciones y respuestas sociales desde cuatro líneas: salud colectiva, educación, producción y conflicto armado/construcción de paz. Todo esto con el fin de subsanar vacíos de información y producir conocimiento pertinente que pueda aportar a la toma de decisiones por parte de las diversas instituciones y organizaciones sociales.

En la línea salud colectiva adaptamos el modelo interpretativo complejo multidimensional (10) de la epidemiología crítica (11) y las discusiones sobre la determinación social de la salud (12), con el fin de superar las miradas limitadas al "pico del iceberg" que desconectan la pandemia de su contexto y separa "los patrones fenotípicos y genotípicos de los contextos, los estilos de vida individuales separados de los modos de vida, los casos de enfermos separados de su pirámide socio-poblacional y los factores de riesgo como asuntos aislados" (13).

La figura 1 conecta las dimensiones individual, particular y general del modelo de subsunción de la epidemiología crítica. En lo individual se incluyen datos epidemiológicos y poblacionales de cada uno de los municipios, que mapeamos e interrogamos en función de las otras dimensiones para reconocer las dinámicas de la ruralidad. En la dimensión particular se incorporan estudios sobre Urabá, que sitúan condiciones propias de la dinámica y prácticas sociales, económicas y culturales, en diálogo con los relatos de integrantes de organizaciones locales. La dimensión 
general es una escala de orden departamental, que permite cruzar la lectura subregional en términos de las lógicas de desarrollo históricamente instaladas en Antioquia. Las tres dimensiones interrogan la lógica antropocéntrica hoy en debate $(5,14)$; cruzan las perspectivas sobre el desarrollo global y local; y focalizan lo veredal/rural en conexión con lo municipal, subregional y departamental, sin descontar su inserción en lógicas globales.

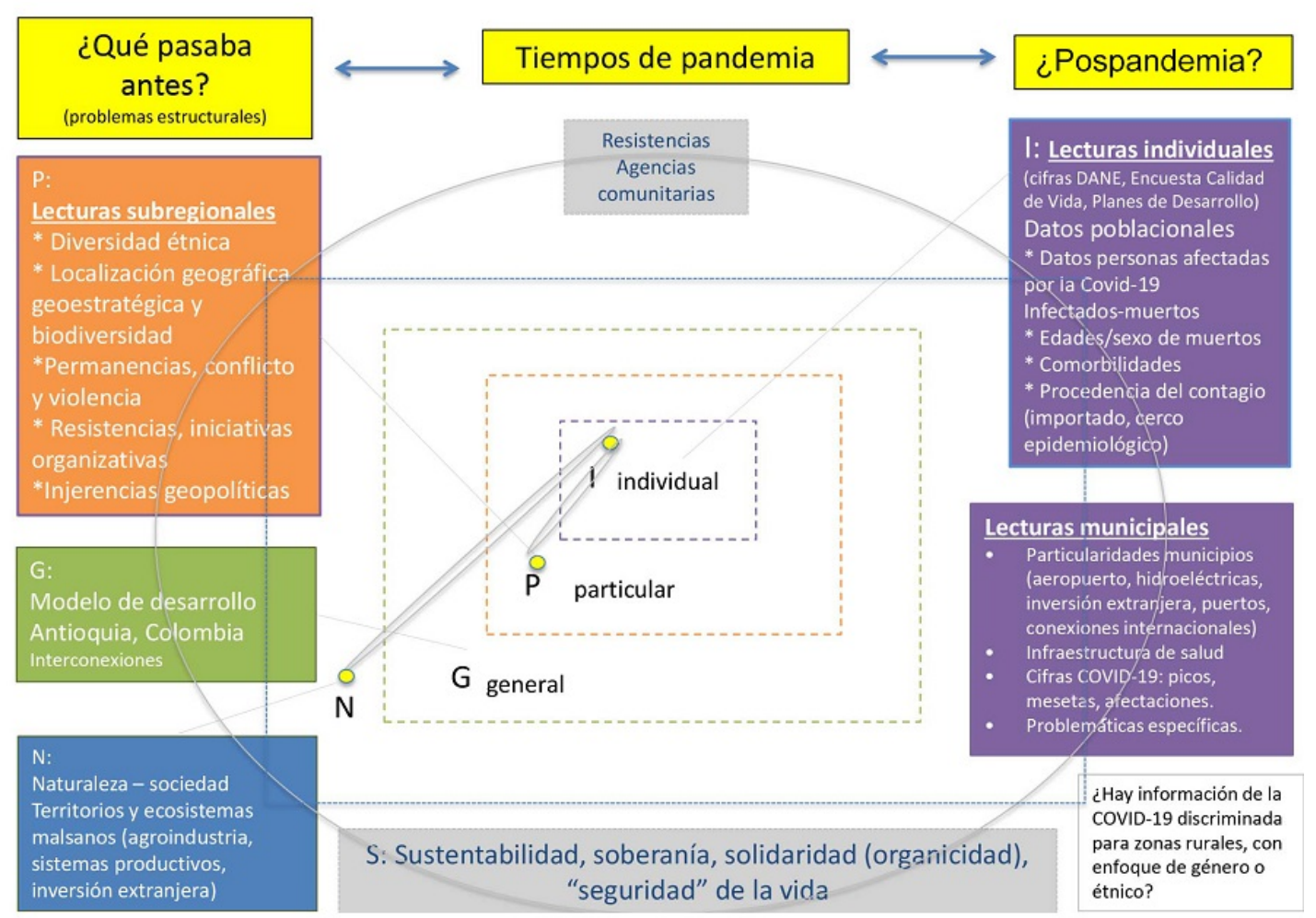

Figura 1 Modelo de análisis línea de salud colectiva Fuente: adaptado de Breilh (10,13).

Se construyó una línea de tiempo para ubicar hitos en la trayectoria de la pandemia, contrastando con intervenciones estatales y experiencias comunitarias. Esta es una lectura espaciotemporal que inicia con la declaración de la pandemia por la OMS hasta noviembre de 2020, incluyendo además elementos particulares de la subregión, para interrogar la información disponible de los once municipios. Ver la figura 2.

La combinación del modelo (figura 1) y la línea de tiempo de la pandemia (figura 2) se denomina Modelo escalar de salud colectiva en tiempos de pandemia. Acudimos a la revisión documental de fuentes secundarias como los datos del Instituto Nacional de Salud (INS), del Departamento Administrativo Nacional de Estadística (DANE), y otros estudios y análisis de la pandemia, además de entrevistas, conversatorios y grupos focales con organizaciones, líderes sociales y comunidad en general. Se utilizaron herramientas tecnológicas y de conectividad remota, con algunas actividades presenciales. 
Beatriz Elena Arias López / Eulalia Hernández Ciro / Alderid Gutiérrez Loaiza

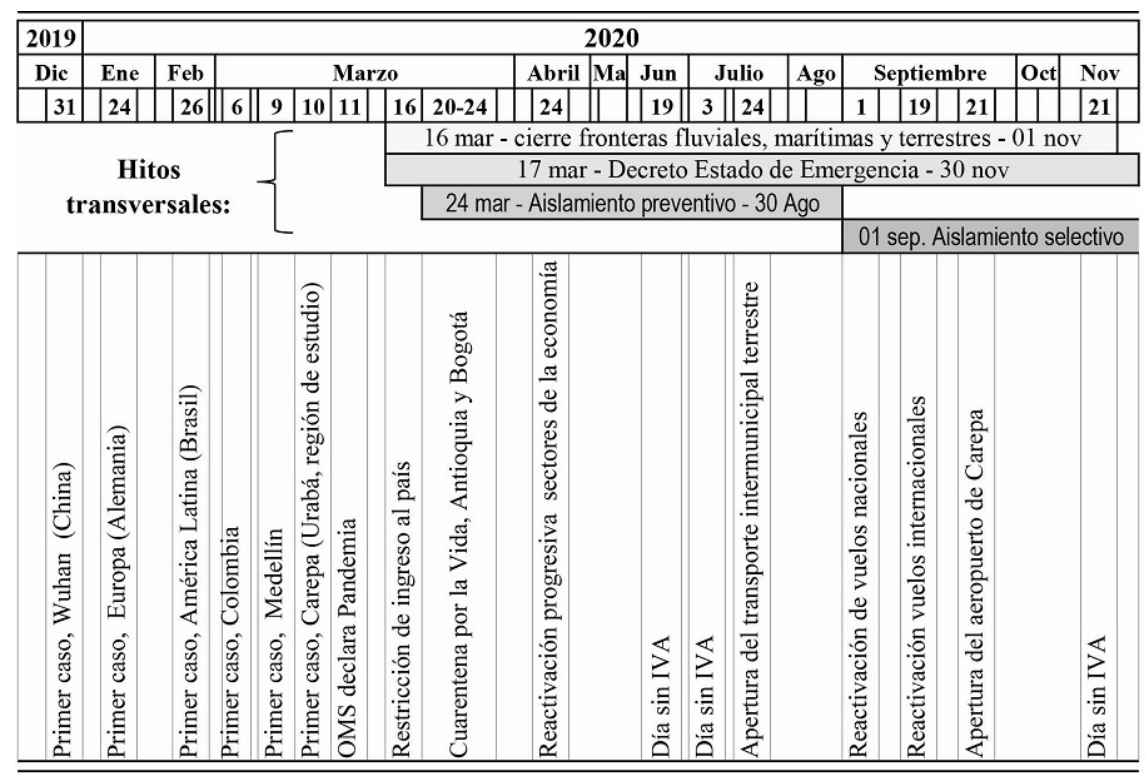

Figura 2 Línea del tiempo. Hitos transversales de salud colectiva Fuente: elaboración propia a partir de la información oficial de (15).

Durante los primeros meses de confinamiento se contactó vía telefónica a líderes de organizaciones con las que teníamos relacionamiento previo para realizar conversatorios, mapeos participativos, ejercicios de etnografía y cartografía social. Esto con el propósito de documentar afectaciones, apoyos, además de estrategias comunitarias y organizativas para hacer frente a la pandemia. Las grabaciones de voz, diarios de campo, fotografías y georeferenciación sirvieron para registrar la información. Se realizaron discusiones al interior del grupo de investigación y se cruzó la información de fuentes documentales a la luz de la información recolectada en campo.

El ciclo de conversatorios denominado Ruralidad y paz: voces de la pandemia desde las regiones, permitió el diálogo con las comunidades, así como entre dependencias de la Universidad de Antioquia. Para el caso de Urabá participaron representantes del Consejo Comunitario Pavarandó de Mutatá, Asociación Campesina de San José de Apartadó (ACASA), Agencia para la Reincorporación y la Normalización (ARN), Comité de Mujer y Género del Área de Reincorporación Grupal San José de León, entre otras. ${ }^{2}$ Igualmente se realizaron ocho recorridos territoriales, ocho entrevistas y tres grupos focales con líderes de ACASA, funcionarios de las alcaldías, la ARN y otras instituciones que hacen presencia en la zona, como el Instituto Popular de Capacitación (IPC), la Pastoral Social y la Universidad de Antioquia sede Urabá. También participaron firmantes del Acuerdo de Paz ubicados en las Nuevas Áreas de Reincorporación (NAR) de Bercuarandó y San José de León en Mutatá y del Espacio Territorial de Capacitación y Reincorporación (ETCR) de Brisas, en Carmen del Darién. La interpretación de la información se realizó con la lógica del análisis etnográfico. 


\section{Resultados}

\section{Lecturas subregionales de Urabá}

El Urabá antioqueño hace parte de un amplio territorio histórico-cultural que comprende porciones de los departamentos de Córdoba, Chocó y Antioquia. La subregión se caracteriza por la abundancia de recursos naturales, la pertenencia al Chocó Biogeográfico, la diversidad de su base sociocultural y económica, las condiciones excepcionales de su ubicación con la conexión que presenta el Golfo con el exterior y la navegabilidad de su caudalosa red hídrica. Sin embargo, el Urabá antioqueño presenta uno de los mayores porcentajes de población con menor nivel de calidad de vida, y ha sido históricamente afectado por la violencia (16).

Sin olvidar la interacción con Córdoba y Chocó, este artículo está centrado en el Urabá antioqueño, una de las nueve subregiones del departamento. Localizada al noroccidente, limita al norte con el mar Caribe formando el Golfo de Urabá, al oriente con el departamento de Córdoba, al occidente con el departamento de Chocó y al sur con la subregión del occidente antioqueño. Como actividades productivas predominan la pesca, la ganadería, la agroindustria, la explotación maderera, la agricultura y el turismo. Los once municipios que lo conforman pueden agruparse en tres sectores: la zona norte que incluye Arboletes, Necoclí, San Juan de Urabá y San Pedro de Urabá, con salida al mar caribe; la zona centro, conformada por Apartadó, Carepa, Chigorodó, Mutatá y Turbo, conocida como eje bananero, donde está asentada gran parte de la población urbana y se concentra la producción agroindustrial; finalmente, la zona del Atrato Medio, que incluye a Murindó y Vigía del Fuerte.

Esta sectorización subregional con su diversidad es importante a la hora de comprender las dinámicas de la pandemia, ya que incluye asuntos como: composición étnica, ubicación estratégica de grandes proyectos de agroindustria e infraestructura con inversionistas extranjeros, biodiversidad, aeropuerto y puerto marítimo, rutas de tráfico de migrantes, superposición de territorialidades de la paz y de la guerra, disputas por el territorio y los recursos, presencia de organizaciones comunitarias de amplia trayectoria, débil cobertura en salud, entre otros.

La subregión de Urabá tiene superpuestas múltiples territorialidades étnicas: un $42 \%$ se reconoce como afrodescendiente, el $4 \%$ como indígena y $53 \%$ como mestiza. Esta composición tiene acentos diferenciales en cada municipio, indicando particularidades desde el punto de vista de las afectaciones, disputas territoriales, riqueza cultural, saberes y prácticas del cuidado y la salud. Esta composición multicultural puede verse en la figura 3. 
Beatriz Elena Arias López / Eulalia Hernández Ciro / Alderid Gutiérrez Loaiza

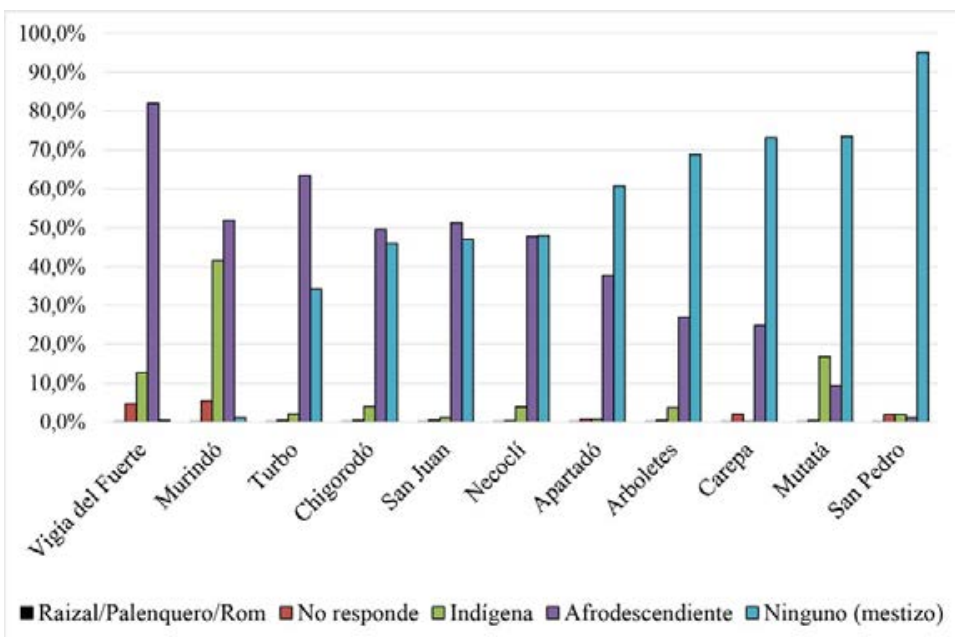

Figura 3 Composición de la población según grupo étnico Fuente: construcción propia a partir de (18).

Turbo es el municipio más poblado (130.191), seguido de Apartadó (127.744) y Chigorodó (59.836). Estos tres municipios están ubicados en el eje bananero, que posee la mayor población en zonas urbanas. El municipio con menos población es Murindó (5.234). Ocho de los once municipios del Urabá tienen mayor población rural, condición relevante a la hora de tener en cuenta las afectaciones por la COVID-19 que se mostrarán más adelante.

Por su condición fronteriza, Urabá es una subregión estratégica para la conexión del país con mercados mundiales. Se espera que entre 2021 y 2022 estén operando tres proyectos portuarios (Puerto Antioquia, Puerto Darién y Puerto Pisisí). A su vez, se espera la conexión de estos proyectos con el interior del país a través de las Autopistas de la Prosperidad (Mar I, Túnel del Toyo, Transversal del Pacífico y demás). Este potencial logístico ha implicado una dinámica de grandes inversiones y expectativa de capitales con presencia de multinacionales en la agroindustria (banano y palma africana), así como explotación maderera. La prensa reseña como antes de la declaratoria de la pandemia se tomaron medidas preventivas de cuarentena en trabajadores del concesionario Autopistas Urabá S.A.S, procedentes de China, que presentaron algunos síntomas relacionados con la COVID-19 (20).

El tapón del Darién, ubicado en la frontera entre Colombia y Panamá, es una zona utilizada como ruta irregular por grupos armados ilegales y tránsito de migrantes. Se calcula que durante 2019 cerca de 24.000 migrantes de 39 nacionalidades buscaron pasar a Centroamérica con destino a Estados Unidos y Canadá. La pandemia agudizó problemas de decenas de migrantes varados en lugares como Necoclí, que, entre otros, tiene dificultades con la cobertura y abastecimiento de agua potable. Ante el cierre de fronteras municipales e internacionales, en agosto de 2020 se reportaron cerca de 350 personas represadas en Necoclí (21), así como en Arboletes y Turbo.

La heterogeneidad del Urabá también es visible en: 
diversos procesos de colonización que ha vivido a lo largo de su historia, en una interacción de éstos con las insurgencias y el paramilitarismo, en el impacto diferencial de la instalación del proyecto agroindustrial bananero en una zona de colonización y en las diferentes respuestas sociales que promovieron sus pobladores para afrontar el conflicto armado (16 p.263).

En esta zona ha existido, desde la década de 1970, una superposición de disputas por los recursos y el control territorial entre guerrillas de las FARC (grupo desmovilizado en 2016) y el Ejército Popular de Liberación. Igualmente, desde comienzos de 1990, hay presencia de grupos de autodefensas, paramilitares $\mathrm{y}$, en las últimas décadas, bandas criminales como el Clan del Golfo. Estas disputas territoriales han generado masacres, homicidios, desplazamientos forzados, asesinato selectivo de líderes sociales, secuestro, extorsión, entre otros. Después del Acuerdo de Paz de 2016, buena parte de los municipios del Urabá y los que comparten frontera con el departamento del Chocó fueron priorizados para el postconflicto. En la actualidad, estas poblaciones tienen aprobados Programas de Desarrollo con Enfoque Territorial (PDET), además de concentrar población excombatiente, ubicada con sus familias de origen en las NAR.

Cabe destacar la presencia de una gran cantidad de organizaciones comunitarias, culturales, deportivas, religiosas, de mujeres, de jóvenes y víctimas, de cooperación internacional, organizaciones no gubernamentales, centros de pensamiento e instituciones universitarias. Estas organizaciones han hecho presencia en la zona, conformando un tejido vital para afrontar las complejidades de la subregión y de la pandemia.

La infraestructura pública de servicios de salud es precaria. Urabá cuenta con once Empresas Sociales del Estado, 164 Instituciones Prestadoras de Salud, clínicas privadas y centros de salud. En Apartadó solo hay 67 camas de cuidado intensivo, distribuidas entre tres clínicas privadas (22). Esto representa un aumento considerable si se tiene en cuenta que al inicio de la pandemia solo tenía 25. Además de atender personas de la subregión, estas clínicas también deben destinarse para remisiones de Córdoba y Chocó. Según cifras de junio de 2020, la subregión contaba con 270 camas de hospitalización (23), lo que implica una cobertura de cinco camas por cada 10.000 habitantes. Muchos casos deben trasladarse a Medellín y su Área Metropolitana o al Oriente antioqueño. La población afiliada al sistema de seguridad social en salud en 2018 para la subregión de Urabá fue del 79,5\% (24), lo cual indica una amplia cantidad de población sin cobertura, sin tener en cuenta temas de acceso y calidad, con importantes brechas en sectores rurales.

Según la Encuesta de Calidad de Vida de 2019, la cobertura residencial de agua potable en términos porcentuales en Urabá es del 60,3 con niveles críticos si se revisan los porcentajes a nivel municipal. Vigía del Fuerte, Murindó y San Juan de Urabá se encuentran entre 0,0 - 21,0; San Pedro, Arboletes y Necoclí entre 21,4 - 43,0; Turbo y Mutatá entre 43,1 - 61,0; Carepa y Chigorodó entre 61,1 - 78,1. Sólo Apartadó se ubica entre 78,2 - 99,8. La subregión presentó el déficit cualitativo de vivienda rural más alto en Antioquia con un 65,7\%, y la penetración de banda ancha para 2019 fue tan solo de 9,1\% (24). 


\section{Voces locales: la pandemia en Urabá}

A continuación, se presentan algunos testimonios relacionados con la situación en los territorios y los procesos organizativos luego de las medidas de confinamiento. Estas voces describen lo que venían haciendo las comunidades para afrontar estas situaciones, y las expectativas, necesidades y propuestas que proyectaban en el corto plazo.

Los participantes manifestaron diversas problemáticas: dificultades para comercializar productos agropecuarios por las limitaciones de movilidad; preocupación por el accionar de grupos armados relacionados con el cierre de fronteras; la restricción de movilidad entre las veredas; y la estigmatización a quienes consultaran los servicios de salud o tuvieran algún síntoma asociado al COVID-19. En general, los participantes mostraron preocupación porque conocían la capacidad limitada de respuesta del sistema público de salud, más aún en las zonas rurales.

Los líderes de Urabá coincidieron en afirmar que las acciones desplegadas por los gobiernos no llegaban a las veredas. Además, se incrementaron los problemas relacionados con la disponibilidad de alimentos. Mientras participaban del conversatorio virtual, uno de los líderes cumplía una función de control del ingreso de personas a su corregimiento, que se condice con la respuesta de los transeúntes, quienes mostraban su cédula a este veedor comunitario. Ante la ausencia estatal se genera la acción comunitaria.

En términos generales, sus preocupaciones -más que por la enfermedad- hacen referencia a la sensación de abandono y desamparo. Esta se incrementó con las diversas restricciones, ya que las entidades e instituciones que tenían programas sociales focalizados dejaron de acompañarlos. Un líder del corregimiento de San José de Apartadó cuenta cómo las comunidades empezaron a generar procesos para mitigar las necesidades de alimentación y cuidado para adultos mayores, personas con discapacidad, mujeres cabeza de familia, entre otros. Una de las estrategias fue la "solidaridad del uno con el otro": hicieron recolectas y trueques; ubicaron casetas en las veredas para quien pudiera compartir lo que tenía e intercambiar productos; en algunos municipios los campesinos donaron su producción a los demás habitantes, sobre todo de zonas urbanas.

Los habitantes sabían que la alimentación sería un asunto vital, así que iniciaron planes de siembra de alimentos de la canasta básica, diversificando cultivos para garantizar la soberanía alimentaria y el fortalecimiento de las unidades productivas. Se buscaron alternativas para comercializar la producción agropecuaria ante las restricciones de movilidad, y se gestionaron transportes y apoyos, al tiempo que se pusieron en marcha mercados móviles campesinos.

Ante el desamparo institucional, se retomaron procesos organizativos para enfrentar la situación. La existencia de estos procesos posibilitó mantener informadas a las comunidades de las medidas de protección y prevención, a través de llamadas telefónicas y de reuniones virtuales, 
cuando la conectividad lo permitía. Es probable que los aprendizajes organizativos de las poblaciones durante el conflicto armado se hayan tornado en estrategias para esta nueva adversidad.

En los conversatorios los participantes manifestaron la preocupación por la situación de los líderes sociales asesinados antes y durante el confinamiento: "mientras las comunidades están confinadas y todo está parado, las preocupaciones son crecientes pues no sabemos lo que pasará con los diferentes proyectos" (25). Igualmente se refirieron a los procesos de titulación de tierras, las acciones de denuncia por proyectos extractivos mineros, la ocupación del territorio por parte de las Autodefensas Unidas de Colombia, y la falta de cumplimiento en la implementación del Acuerdo de Paz por parte del gobierno nacional.

\section{Lo que dicen los datos}

Tomando como referente la fecha del primer caso nacional se identificó el primer caso reportado en cada municipio. Carepa registró el suyo a los cuatro días, seguido de Apartadó el 14 de marzo. El 11 de abril apareció en San Pedro de Urabá y solo hasta mayo se presentó en Turbo y Arboletes, el 9 y 10 respectivamente, cerrando en Chigorodó el 27 y en Vigía del Fuerte el 30. En junio se reportó el primer caso en los cuatro municipios restantes: el 1 en San Juan de Urabá, el 10 en Murindó, el 12 en Necoclí y el 19 en Mutatá. Después de este primer caso, solo en municipios como San Pedro de Urabá se registraron nuevos casos tres meses después, en Carepa un mes y medio después, mientras en Arboletes y San Juan de Urabá, aparecieron a los 15 días (figura 4). En el resto de municipios, después del reporte del primer caso, se presentaron más reportes inmediatamente.

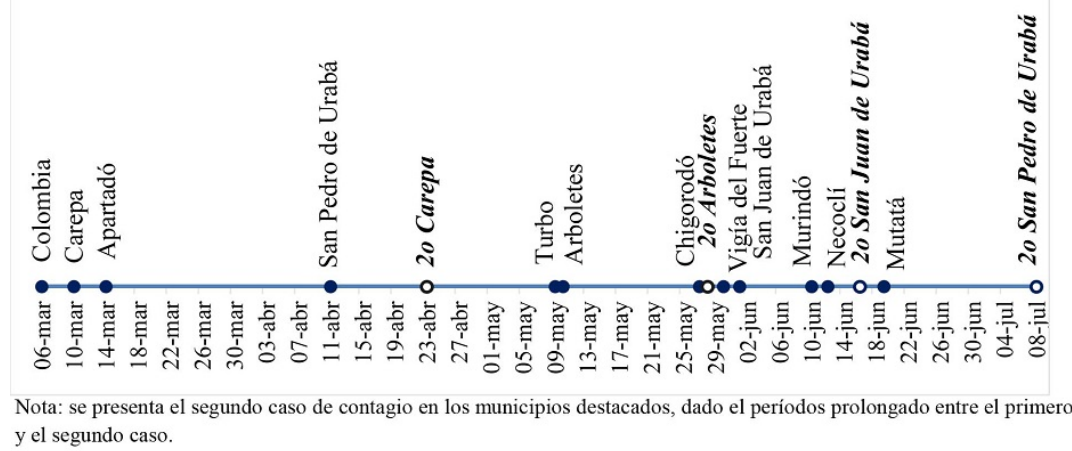

Figura 4 Primer caso de COVID-19 reportado en cada uno de los municipios de Urabá Fuente: construcción propia con base en INS (26).

Con el fin de explorar la velocidad de propagación del COVID-19, se tomó como referencia la fecha del primer caso en cada municipio. ¿Cuántos días tardó en presentarse el caso número 100 de contagio?

Tomando datos acumulados, Turbo fue el de más rápido crecimiento ( 23 días en llegar al caso 100); Necoclí y Chigorodó tardaron 27 y 29 días; mientras San Pedro de Urabá tardó 116 días. En los municipios de Murindó, Vigía del Fuerte y San Juan de Urabá, hasta el momento de corte 
(noviembre 24 de 2020), no se había llegado a 100 casos, mostrando cifras totales de 85, 68 y 53 , respectivamente.

Apartadó es el municipio con la mayor cantidad de contagios (3.872), con una tasa de aproximadamente 30 por cada mil habitantes, le sigue Turbo con 1.371 casos registrados (figura 5), aunque con una tasa menor, al igual que San Pedro de Urabá. Al contrario, la tasa es especialmente elevada en Murindó, que ocupa el tercer lugar, con 16 casos por mil habitantes, después de Carepa, que ocupa el segundo lugar con alrededor de 26.

El contagio del COVID-19 ocurre en mayor medida en la población masculina, con porcentajes que oscilan entre 51,5\% (Vigía del Fuerte) y 69,3\% (Carepa). Solamente en el municipio de Arboletes los casos son superados por las mujeres con un 51,1\%. En los once municipios, los casos se concentran en personas de 20 a 49 años, especialmente en el rango de 30 a 39 años. En el municipio de Carepa, el mayor número de casos se concentra en edades entre los $20 \mathrm{y}$ 29 años, mientras que en Arboletes y Mutatá la edad de mayor contagio está entre los 40 y 49 años. Otra constante es que el menor número de casos ocurre en las personas más longevas, por encima de $\operatorname{los} 80$ años.

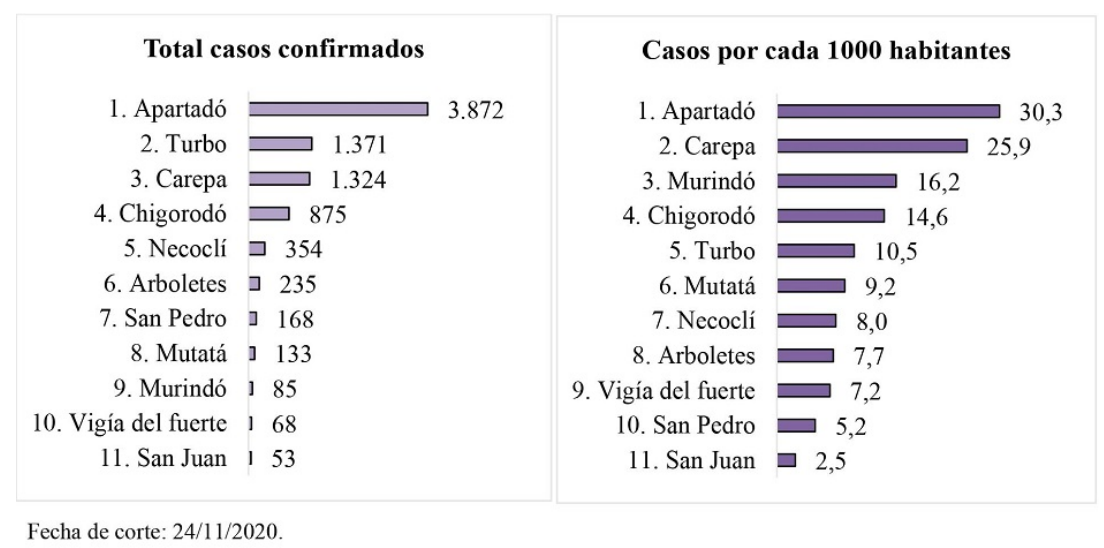

Figura 5 Total de casos de COVID-19 y casos

por 1.000 habitantes en los municipios de estudio

Fuente: construcción propia con base en INS (26).

La mayor letalidad se encuentra en San Juan de Urabá, donde mueren entre 9 y 10 personas de cada cien contagiados (figura 6). Este es un dato llamativo ya que puede ser enmascarado por el menor número de casos acumulados en dicho municipio. Una situación similar se presenta en Necoclí, donde mueren 7 de cada 100 contagiados, aunque en número de casos acumulados ocupa un quinto lugar. Al contrario, las tasas de letalidad en Chigorodó, Carepa y Apartadó oscilan entre 3 y 4 fallecidos por cada 100 contagiados. Esto contrasta con el alto número de casos acumulados, que, como se mostró anteriormente, los ubica en los primeros lugares en el comparativo de estos municipios. Murindó reportó 133 contagios y ningún fallecimiento hasta la fecha de corte. 
Según el perfil de letalidad por sexo fallecen más hombres que mujeres, en un porcentaje que va entre 50 y $80 \%$. Los porcentajes más altos de hombres fallecidos se encuentran en Carepa y Mutatá con un $67 \%$, Arboletes con un 79\% y San Juan de Urabá con un $80 \%$. Ahora bien, pese a que los mayores contagios ocurren en edades tempranas, las muertes se presentan, en mayor medida, en la población superior a los 60 años $(60 \%)$. En forma particular, en Turbo y Vigía del Fuerte, los mayores porcentajes de muertes aparecen desde los 50 años, mientras en San Juan de Urabá desde los 40 años.

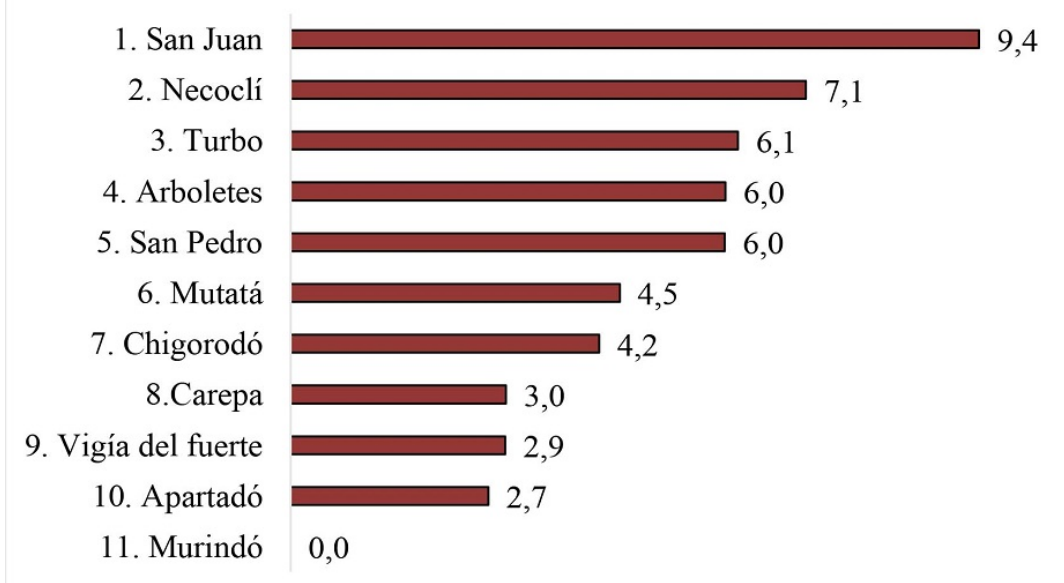

Fecha de corte: noviembre 24 de 2020

Figura 6 Porcentaje de letalidad del COVID-19 en los municipios de estudio Fuente: construcción propia con base en INS (26).

Como se observa en la figura 7, el ejercicio del impacto de los hitos (primer y segundo día sin IVA, y apertura del transporte terrestre) se realizó revisando los casos presentados dos semanas después, en una ventana de revisión de cuatro días. Es decir, se observó cómo se comportaron los contagios en los días 12, 13, 14 y 15, después de sucedido el hecho, y se tomó un promedio en la tasa de crecimiento de esos días. Frente al primer día sin IVA, los municipios en donde más aumentaron los casos de contagio fueron Chigorodó y Mutatá. En relación con el segundo día sin IVA, los contagios aumentaron llamativamente en Mutatá, Carepa, Necoclí, Murindó, Chigorodó y Arboletes. Los cambios más sensibles se presentaron luego de la apertura del transporte terrestre, particularmente en Necoclí, Mutatá, Carepa y Arboletes. Estos hitos no tuvieron efectos en los municipios de Vigía del Fuerte, San Juan de Urabá y San Pedro de Urabá. Se advierte que los contagios pueden aumentar por múltiples factores. Los hitos intentan mostrar una correlación, que desde luego no es determinante, por lo que se sugiere identificar las posibles causas en los aumentos de contagios, en las fechas señaladas, y contrastarlos con las vivencias y experiencias de los lugareños. 


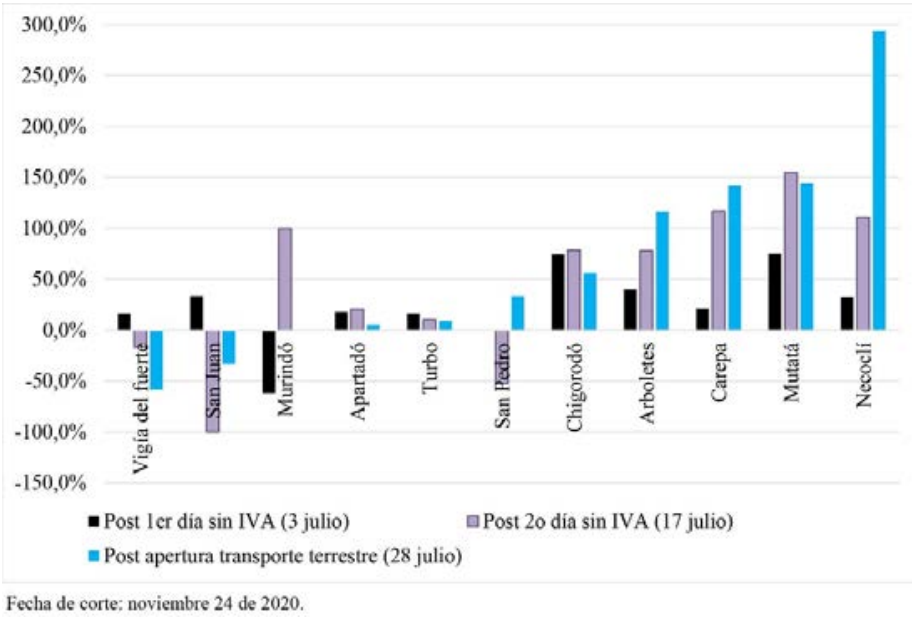

Figura 7 Promedio de crecimiento en los casos de contagio, dos semanas después de algunos hitos Fuente: construcción propia con base en INS (26).

\section{Discusión. Interrogar los datos}

La mayor limitación del presente estudio ha sido la ausencia de datos diferenciados para las áreas rurales, procedencia étnica y precisión en el origen del contagio (reporte "en estudio"). Según el INS, la información étnica es responsabilidad de las autoridades locales del país. Su calidad depende del correcto diligenciamiento de los profesionales de salud que notifican según el autorreconocimiento de las personas. Esta ausencia de datos diferenciados significa un vacío importante a la hora de comprender la complejidad de las afectaciones y emprender acciones. La mayoría de los análisis espaciotemporales y proyecciones epidemiológicas publicados en Colombia se han realizado en centros urbanos, bajo una lógica no diferenciada $(27,28)$, con algunas excepciones reflexivas, sobre regiones periféricas y con diversidad étnica $(29,30)$. Esto nos lleva a posicionar nuestras preguntas y rutas de exploración como prioridades para orientar la respuesta a la pandemia de COVID-19 en América Latina (31). Al combinar la línea de hitos claves en la subregión, el modelo escalar coincide en su lógica con trabajos realizados en Argentina, donde también se identificaron momentos diferenciados de la pandemia y de su gestión. En estos estudios se señalan desarrollos normativos, comunicacionales e iconográficos particulares, indicando ausencias y silencios frente a grupos incluidos y excluidos (32).

En esta misma línea se encuentran iniciativas como las de la Oficina de las Naciones Unidas para la Coordinación de Asuntos Humanitarios, que realiza un ejercicio mensual para informar el Impacto humanitario por la COVID-19; el Observatorio COVID-19 en América Latina y el Caribe de la Cepal, en donde, además de las variables económicas y sociales tradicionales, se hace seguimiento a las medidas de cuarentena impuestas por los gobiernos nacionales, las disposiciones hospitalarias con instalaciones UCI y el proceso de vacunación (33); el Observatorio social del coronavirus "Pensar la pandemia", del Consejo Latinoamericano de Ciencias Sociales CLACSO (6), que se ha dedicado a documentar asuntos relacionados con 
educación y cultura, género, raza/etnia, territorio y movilidad, violencias, procesos políticos y sociales, entre otros.

Los datos empíricos de este estudio muestran una rápida aparición de casos en dos municipios del eje bananero: Carepa y Apartadó. En Carepa esto se podría explicar por la presencia del Aeropuerto Antonio Roldán Betancur. Por otro lado, la contigüidad y dinámicas de relacionamiento entre Carepa y Apartadó hacen que la frontera entre ambos municipios sea un artificio. Estos dos municipios, junto con Turbo, son el epicentro económico de la subregión lo que implica mayor movilidad. Sin embargo, dichas poblaciones son las que tienen menores índices de pobreza y mayor infraestructura para acceder a servicios de salud y de diagnóstico. En el caso de San Pedro de Urabá, la aparición precoz de casos podría relacionarse con la situación del tránsito de migrantes, asunto también señalado por la Oficina de la Organización de Naciones Unidas para la Coordinación de Asuntos Humanitarios (34). Esto posiciona la relación migraciones y COVID-19 como un tema relevante en investigación (35-37).

Los municipios que más tardaron en tener casos positivos fueron los del Atrato Medio. Al tener grandes limitaciones de accesibilidad, estuvieron más protegidos frente a los contagios, aunque en términos de letalidad están entre los más afectados. En cuanto a la velocidad de propagación, teniendo como criterio el número de días que se tardó en llegar de uno a 100 casos, los municipios que presentaron el primer caso en forma temprana fueron paradójicamente los que más tardaron en llegar al número 100, mientras que los municipios que presentaron casos tardíamente, aceleraron el contagio. Los municipios más "integrados” y conectados han recibido mayores servicios de atención y disposición a tomar medidas de cuidado y seguridad. Esto se debe a que las actividades laborales que concentran mayor población pudieron tener medidas más formalizadas, mejores infraestructuras de servicios públicos y de acceso a los servicios de salud. Esto no ocurre en sectores alejados, caracterizados por la informalidad en las dinámicas de trabajo, débil infraestructura y limitación en el acceso a los servicios de salud. El caso de Turbo es llamativo porque perteneciendo al eje bananero exhibe el crecimiento más acelerado desde el caso 1 al caso 100 (23 días). Además, el rango de edad de los fallecimientos se concentra en edades tempranas. La dinámica portuaria, legal e ilegal, podría ser un elemento para explorar.

El número de casos acumulados guarda la misma tendencia de los datos de población de los municipios, es decir, el mayor número de casos se da en los municipios con mayor número de población. Sin embargo, se encuentra que un municipio como Murindó que ocupa el noveno lugar en casos absolutos, asciende al tercer lugar en tasas por mil habitantes. Nuevamente encontramos mayor afectación en los municipios más excluidos de la subregión.

La distribución por sexo estaría mostrando la relación de los trabajos habitualmente asignados con el género de la persona, así como una mayor exposición para hombres que para mujeres. Esto puede leerse en un municipio como Carepa, donde además de su cercanía a la agroindustria, se cuenta con un batallón del ejército. Estos ámbitos son de concentración de población especialmente masculina. 
Revisando la letalidad surgen preguntas relacionadas con desigualdades históricamente acumuladas. Los indicadores de pobreza del municipio de San Juan de Urabá, según la Encuesta de Calidad de Vida 2019 del Departamento de Antioquia señalan que

el porcentaje de personas en situación de pobreza y en condición de indigencia muestran valores altos, pues la incidencia de la pobreza llega al $62 \%$ y la de indigencia casi al $30 \%$. Los hogares en condición de pobreza por NBI llegan al 38,5\% del total. Estos valores evidencian una alta vulnerabilidad material de las personas en este municipio para afrontar la pandemia y sus consecuencias (38 p.8).

Esta situación presenta un dato paradójico en un municipio como Murindó, donde a la fecha de corte no se registraba ningún fallecimiento, dejando interrogantes sobre la ausencia o error en el sistema de información y/o particularidades de este municipio, el cual presenta una composición étnica que combina en proporciones semejantes la población afrodescendiente e indígena. Lo anterior contrasta con la más baja letalidad en municipios como Chigorodó, Carepa y Apartadó, donde los indicadores de pobreza multidimensional son los más bajos. Estos hallazgos son coincidentes con algunos análisis en Brasil, donde se señala la forma desigual de presentación del COVID-19, especialmente en cuanto al número de víctimas mortales, y su relación de clase y etno-racial, con expresiones diferenciales en la ciudad y el campo (39).

Respecto a los hitos priorizados en la metodología, días sin IVA y apertura de vías intermunicipales, parecen no afectar en forma significativa municipios como Vigía del Fuerte o Murindó, no así a los municipios del eje bananero y los del norte. La decisión del gobierno nacional de apoyar estas medidas envió un mensaje contradictorio a la población que se tradujo en que quizás no era necesario cuidarse, y que era posible disminuir las medidas de protección. Municipios como Turbo, que tienen un puerto marítimo, fueron más sensibles a las aperturas económicas, con un aumento notable de casos. Otro tanto sucedió en municipios cercanos que, si bien no tienen el mismo nivel de integración, pudieron ser sensibles al mensaje contradictorio de los días sin IVA. Municipios del eje bananero y del norte en general fueron muy sensibles a la apertura del transporte terrestre, con aumento de casos relacionados. Recuérdese que estos últimos se asocian con el tránsito de migrantes y de turistas, además de su conexión con Córdoba.

Estas dinámicas diferenciadas en la subregión resaltan la importancia de desarrollar comprensiones complejas. La información empírica presentada muestra que los datos epidemiológicos por sí solos y las estrategias estandarizadas basadas en medidas de protección de responsabilidad individual, sin considerar asuntos de inequidad estructural, justicia social y respuestas sociales, pueden tener grandes limitaciones (40), ocultando información determinante. Citando a Tetelboin et al. (41): "estas intrusiones controladoras en las vidas de las comunidades, las familias y las personas tienden a distribuirse desigualmente por edad, situación socioeconómica y lugar de residencia" (41 p.13). Esto exige analizar los datos epidemiológicos como expresión decantada de dinámicas económicas, políticas, ideológico- 
culturales y ambientales, que también tienen su correlato en percepciones sobre la enfermedad, imaginarios sobre la pandemia y acciones comunitarias y territoriales, como lo venimos documentando en el Observatorio.

Lo que logramos identificar en el trabajo de campo fue el despliegue de prácticas de cuidado, individuales y colectivas, donde se conjugaron saberes sobre el uso de plantas medicinales, automedicación, fortalecimiento de producción de pancoger, intercambios, trueques, donaciones y autogestión territorial de la pandemia por parte de líderes y comunidades. Se combinaron allí los mandatos de bioseguridad individual, con medidas actualizadas desde sus memorias y aprendizajes derivadas de la vivencia del conflicto armado, relacionadas con el confinamiento y las medidas de control, además de un sinnúmero de respuestas de resistencia. A pesar de las contradicciones, el miedo y la incertidumbre, cabe resaltar la valoración de la ayuda mutua y la cooperación en tiempos de crisis, reclamado en otros análisis similares (42). Estos aspectos deberían también considerarse en la comprensión y manejo de la pandemia.

\section{A manera de cierre}

A la hora de documentar los contagios y muertes causadas por la COVID-19, las cifras e indicadores oficiales emitidas por el INS y otras instituciones competentes son una importante herramienta para cuantificar la pandemia y tomar medidas para mitigar sus impactos. Pero es necesario interrogar estas cifras y redimensionarlas para conocer las múltiples afectaciones. Para ello propusimos el Modelo escalar de salud colectiva en tiempos de pandemia, que incorpora el modelo de análisis desde la salud colectiva y la problematización espaciotemporal.

Las cifras municipales del Urabá antioqueño, interrogadas en clave de las dinámicas subregionales y las voces y vivencias de los pobladores y organizaciones, permiten generar algunas reflexiones respecto a los desafíos de equidad en materia de salud y bienestar de las poblaciones. Estas se vieron agravadas por las vulnerabilidades tradicionalmente existentes y las emergentes por la COVID-19.

A la problematización de los datos, subyace el marcado contraste entre integración/exclusión de los once municipios, así como la superposición de lógicas de legalidad e ilegalidad presentes en sus territorios. Urabá es una de las regiones más complejas del departamento y del país, donde se superponen brechas históricas en temas de salud, educación, alimentación y servicios públicos, entre otros. En la subregión se dan varios procesos de transformación urbana, proyectos de infraestructura, escenarios de reincorporación, superposición de ordenamientos e institucionalidades para la paz, que se desarrollan simultáneamente con la presencia y agencia de grupos armados ilegales y bandas criminales. ¿Hasta dónde es posible documentar, pero además incidir sobre dinámicas y decisiones de tan múltiples y diversos actores? En este contexto parece difícil tener un nivel de gobernanza efectiva.

Este estudio sugiere que las medidas epidemiológicas no pueden desoír a los pobladores ni desconocer los espacios rurales como productores de saberes. Hay una pluralidad en función 
del género, de la intergeneracionalidad, y de las identidades étnicas y territoriales (comunidades campesinas, afro, indígenas, mestizas, anfibias, pescadoras y excombatientes). Estas últimas han vivido históricamente dinámicas de exclusión y, por tanto, requieren una atención diferenciada para el goce efectivo de sus derechos. La vulnerabilidad social es un factor importante en la atención a los efectos indirectos de la cuarentena: a mayor vulnerabilidad social índices de pobreza y necesidades básicas insatisfechas- mayores impactos. Súmese a ello el aumento de asesinatos y amenazas a líderes sociales, los toques de queda ejercidos por grupos delincuenciales, y su efecto en el temor en consultar por retaliaciones de los grupos armados.

Ante la ausencia de respuestas institucionales, las comunitarias no se hacen esperar. Los pobladores organizados que han aprendido a resistir, en medio de tan diversos intereses en pugna, recogen sus trayectorias y aprendizajes. Hoy, nuevamente, en situación de pandemia, asumen el liderazgo, no solo para poner en práctica, garantizar y ser veedores de las medidas de protección y cuidado, sino también para mejorar las condiciones de subsistencia. La COVID-19, más que una enfermedad, es un hito que revela nuevamente los desbalances estructurales que existen en el territorio.

Frente a los horizontes investigativos abiertos y los propósitos del Observatorio cabe recalcar la importancia de los análisis complejos: miradas interdisciplinarias, intercambios entre la epidemiología y las ciencias sociales, y el diálogo de saberes con los habitantes de los territorios. Este tipo de análisis permite ver más allá de la 'punta del iceberg', traducida en cifras y datos descontextualizados. El propósito será enriquecer las miradas y co-producir un conocimiento social pertinente, que aporte insumos para la toma de decisiones de instituciones públicas, privadas y organizaciones sociales, y que ayude a superar la crisis que vivimos, agudizada por esta pandemia.

\section{Consideraciones éticas}

Esta investigación no requirió aval ético, sin embargo, en el proceso se tuvieron en cuenta los resguardos éticos en el acercamiento con las comunidades.

\section{Contribución de los autores}

Los tres autores realizaron aportes a la idea y diseño del estudio, recolección de datos, análisis e interpretación. Igualmente, todos participaron en la redacción y elaboración de la versión final del artículo entregado a la Revista.

\section{Financiación}

La investigación "Observatorio ruralidad y paz en contextos de pandemia y postpandemia" fue financiada por el Fondo UdeA responde al COVID-19, de la Vicerrectoría de Investigación de la Universidad de Antioquia, y fue cofinanciada por el Instituto de Estudios Regionales 
y las Facultades de Enfermería, de Educación y de Ciencias Económicas de la Universidad de Antioquia. Contó con el apoyo de la Corporación de Estudios, Educación e investigación Ambiental (CEAM). Acta N²020-37010 del 15 de septiembre de 2020.

\section{Conflictos de interés}

Los autores declaran no tener conflictos de interés.

\section{Referencias}

1. Neira-Rozas J. Ciencias Sociales y Salud. Pre-textos para el Debate. Revista Austral de Ciencias Sociales. 2001;5:127-138. https://doi.org/10.4206/rev.austral.cienc.soc.2001.n5-11

2. Arias-López BE, Torres-Marín B. Veinte años construyendo la salud colectiva. La experiencia de la Maestría en salud Colectiva de la Universidad de Antioquia. Cuadernos del Doctorado. 2017;18:71-94. http://www.doctoradosaludp.unal.edu.co/wp-content/uploads/2018/04/Cuaderno-18 .pdf

3. Nunes ED. Paradigmas de la salud colectiva: breve reflexión. Salud Colectiva. 2014;10(1):57-65. https ://doi.org/10.18294/sc.2014.208

4. Liborio M. ¿Por qué hablar de salud colectiva? Rev Méd Rosario. 2013;79:136-141. https://doi.org/10. 12804/revistas.urosario.edu.co/revsalud/a.6123

5. De Sousa SB. La cruel pedagogía del virus. Buenos Aires: CLACSO; 2020.

6. CLACSO. Pensar la Pandemia. Observatorio Social del coronavirus; 2021 https://www.clacso.org/pens ar-la-pandemia-observatorio-social-del-coronavirus/

7. Quijano O, Corredor C. Pandemia al sur. Buenos Aires: Prometeo Libros; 2020.

8. Robledo C. La vejez. Reflexiones de la postpandemia. Medellín: Opción Colombia; 2020.

9. Piazzini-Suárez CE. Los mapas y calendarios de la pandemia. Geopolítica(s). Revista de Estudios sobre Espacio y Poder. 2020;11(Especial):265-274. https://doi.org/10.5209/geop.69351

10. Breilh J. Critical epidemiology and the People's health. New York: Oxford University Press (in-print).

11. Breilh J. Epidemiología crítica. Ciencia emancipadora e interculturalidad. Buenos Aires: Lugar Editorial; 2003.

12. Morales C, Eslava JC. Tras las huellas de la determinación. Bogotá: Universidad Nacional de Colombia; 2015.

13. Breilh J. La salud colectiva en tiempos de pandemia: una nueva visión y metodología crítica de la pandemia. Quito: Universidad Andina Simón Bolívar; 20 Abril de 2020. https://www.youtube.com/ watch? $\mathrm{v}=\mathrm{Gt} 6 \mathrm{O} 7 \mathrm{ebxif4 \& ab \_ channel=AndinaVirtual}$

14. Sánchez G, Blaustein M, Rascovan N. Pandemias de ayer y hoy. Del antropoceno al antro obsceno. Revista Anfibia. 2020; http://revistaanfibia.com/ensayo/del-antropoceno-al-antro-obsceno/?fbclid=I wAR0cOO5Klm9BvbhlbIb_FboGFaFRhjPyNSxZ8KaNbP9qrhiklaE00czGIPQ 
15. República de Colombia. Acciones tomadas por el Gobierno. Bogotá: Gobierno nacional. https://coron aviruscolombia.gov.co/Covid19/index.html

16. García CI, Aramburo CI. Geografías de la guerra, el poder y las resistencias. Oriente y Urabá antioqueños. Medellín: ODECOFI-CINEP-INER; 2011.

17. DANE. Pobreza y desigualdad. Bogotá: Gobierno Nacional. https:/geoportal.dane.gov.co/servicios/se rvicios-web-geograficos/sociedad/pobreza-y-desigualdad/

18. Gobernación de Antioquia. Anuario Estadístico de Antioquia; 2018. Medellín: Departamento Administrativo de Planeación. http://www.antioquiadatos.gov.co/index.php/anuario-estadistico-201 8

19. DANE. Proyecciones de Población. Bogotá: Gobierno Nacional. https://www.dane.gov.co/index.php/e stadisticas-por-tema/demografia-y-poblacion/proyecciones-de-poblacion

20. Por prevención, tres ciudadanos chinos en cuarentena en Antioquia. Medellín. El Tiempo. $2020 \mathrm{https} / /$ www.eltiempo.com/colombia/medellin/en-antioquia-tres-chinos-estan-en-cuarentena-p or-posible-coronavirus- 457678

21. Álvarez-Arango L. Migrantes transcontinentales, drama que agudiza en Urabá. Periódico Alma Máter, Medellín: 2020;agosto(699). https://bit.ly/3aDCbsV

22. Minsalud. Capacidad instalada para la prestación de servicios de salud, Sispro. Bogotá: Ministerio de Salud y Protección Social. https://minsalud.maps.arcgis.com/apps/opsdashboard/index.html\#/5985c da246fa4eea83638adac9c253ab

23. Universidad de Antioquia. Guía base para la reactivación económica - Apartadó. Medellín: Universidad de Antioquia, IDEA, Gobernación de Antioquia; 2020. https://bit.ly/36mBken

24. Gobernación de Antioquia. Plan de Desarrollo Departamental. 2020-2023, Medellín: Gobierno Departamental. https://plandesarrollo.antioquia.gov.co/

25. Universidad de Antioquia. Sin tapar bocas. Voces de la pandemia desde las regiones. Análisis de impactos y riesgos en los territorios, el proceso de paz y otros procesos sociales; 2020. https://www .youtube.com/watch?v=R_UUUGRWm_g\&t=1s

26. Instituto Nacional de Salud (INS). COVID 19 en Colombia. Bogotá: Gobierno Nacional. https://www .ins.gov.co/Noticias/Paginas/coronavirus-filtro.aspx

27. Granada-Echeverri M, Molina-Cabrera A, Granada-Echeverri P. Proyección espacio-temporal del COVID-19 en Pereira. Tecnológicas. 2020;23(49):129-146. : https://doi.org/10.22430/22565337.16 55

28. Ruiz-Pérez JI, Aparicio Barrera J. Análisis espacio-temporal del incumplimiento de normas legales sobre el confinamiento en Colombia por COVID-19. Revista Logos, Ciencia \& Tecnología. 2020;12(3):20-32. https://doi.org/10.22335/rlct.v12i3.1251

29. Hurtado-Ortiz A, González-Morales, EC, Licht-Ardila M. Impacto de la pandemia por COVID-19 en el Amazonas, Colombia. Revista de la UIS. 2020;52(2):187-189. https://revistas.uis.edu.co/index.p $\mathrm{hp} /$ revistasaluduis/article/view/11253/10940 
Salud colectiva en tiempos de COVID-19. Modelo escalar para comprender las afectaciones en la ruralidad de Urabá, Antioquia, Colombia, 2020

30. Sandoval EA, Capera JJ. La resistencia sociocultural de la Guardia Indígena del Tolima, Colombia: narrativas subalternas en tiempos del COVID-19. Espacio Abierto. 2020;29(4):209-229. https://dial net.unirioja.es/servlet/articulo?codigo $=7738559$

31. Cucunubá ZM. Investigación científica prioritaria en Latinoamérica para orientar la prevención y el control de la COVID-19. Biomédica. 2020;40(2):9-13. https://revistabiomedica.org/index.php/biom edica/article/view/5882

32. Bernasconi M, Romero MA, Golovanevsky L. Mapeo de políticas públicas locales en Jujuy en contexto de pandemia. Trabajo y Sociedad. 2021;21(36):203-230. http://www.redalyc.org/articulo.oa?id=387 366077010

33. Comisión Económica para América Latina y el Caribe (Cepal). Observatorio COVID-19 en América Latina y el Caribe Impacto económico y social; 2021. https://www.cepal.org/es/temas/covid-19

34. Oficina de las Naciones Unidas para la Coordinación de Asuntos Humanitarios Colombia: Impacto humanitario por el COVID-19. Informe de Situación No. 15, 2020; https://reliefweb.int/sites/reliefw eb.int/files/resources/11082020_colombia_informe_de_situacion_no_12_impacto_covid-19_vf.pdf

35. Rocha JL. La migración centroamericana a los Estados Unidos en tiempos del COVID-19. REMHU 2020;28(60):109-126. https://doi.org/10.1590/1980-85852503880006007

36. Mora-Izaguirre C. La marcha de los flujos mixtos por Costa Rica a la luz de algunas teorías que describen las migraciones del siglo XXI. REMHU 2020;28(60):95-108. https://doi.org/10.1590/1980-858525 03880006006

37. Fernández-Niño JA, Cubillos-Novella A, Bojórquez L, Rodríguez M. Recommendations for the response against COVID-19 in migratory contexts under a closed border: The case of Colombia. Biomédica. 2020;40(2):68-72. https://doi.org/10.7705/biomedica.5512

38. Universidad de Antioquia. Guía base para la reactivación económica - San Juan de Urabá. Medellín: Universidad de Antioquia, IDEA, Gobernación de Antioquia; 2020.

39. Tosta SS. Relação campo cidade em tempos de pandemia. GEOPAUTA. 2020;4(2):245-266. https://d oi.org/10.22481/rg.v4i2.6101

40. Anigstein M, Burgos S, Medina S, Pesse-Sorensen K, Espinoza P, Toledo C. Desafíos y aprendizajes para la promoción de la salud durante la pandemia de la COVID-19 en Chile. Un análisis de experiencias locales desde la salud colectiva. Global Health Promotion; 2021. https://doi.org/10.1177/17579759 20986700

41. Tetelboin C, Iturrieta D, Schor-Landman C. América Latina. Sociedad, política y salud en tiempos de pandemia. Buenos Aires: CLACSO, Universidad Autónoma Metropolitana, Universidad Veracruzana, Universidad Mayor de San Simón, Universidad de Valparaíso; 2021.

42. Springer S. Caring geographies: The COVID-19 interregnum and a return to mutual aid. Dialogues in Human Geography. 2020;10(2):112-115. https://doi.org/10.1177/2043820620931277

Notas

1 Para conocer más sobre esta estrategia, consultar: https://bit.ly/31nDrM4

2 La grabación de este conversatorio está disponible en: https://bit.ly/3wtfj8Q 